CERN-TH/96-360

hep-th/9701075

\title{
Fermion Exchange between D-instantons
}

\author{
J. L. F. BARBÓN \\ Theory Division, CERN \\ 1211 Geneva 23. Switzerland \\ barbon@mail.cern.ch
}

\begin{abstract}
We define fermionic collective coordinates for type-IIB Dirichlet instantons and discuss some effects of the associated fermionic zero modes within the dilute gas framework. We show that the standard rules for clustering of zero modes in the dilute limit, and the fermion-exchange interactions follow from world-sheet Ward identities. Fermion exchange is strongly attractive at string-scale distances, which makes the short-distance Hagedorn singularity between instantons and anti-instantons even stronger.
\end{abstract}

December 1996 


\section{Introduction}

The Dirichlet-brane construction of Ramond-Ramond solitons [1], [2] allows a rather explicit characterization of the weak coupling quantization of such objects. The collective dynamics of a $p$-brane consists of a world-volume interacting theory of open strings propagating in $p+1$ dimensions, and the description of the interactions with the bulk dynamics is equally explicit in terms of the standard coupling between open and closed strings. A special role is played by the Dirichlet instanton of the type-IIB theory, the case $p=-1$ localized in all space-time directions. The "world-volume" is a point, and the collective dynamics is given by a finite-dimensional integral over the multi-instanton moduli space, the integrand being determined by a "zero-dimensional" open string theory. Besides the simpler collective dynamics, D-instantons exhibit a number of special properties, as compared to the rest of the D-branes. It is well known that perturbative string amplitudes in the instanton background are power-behaved at high energy [3], thus introducing "field theoretical" features in string theory. The kind of logarithmic divergences responsible for the world-volume dynamics in the higher branes (via a Fischler-Susskind mechanism), are cancelled in this case by the instanton gas combinatorics, which also ensures the standard clustering properties [4], 河].

The analogue of the static interaction potential for higher branes is the interaction action of D-instantons. The leading-order contribution in string perturbation theory is the cylinder diagram with two Dirichlet boundaries. In terms of massless background fields [6] it corresponds to the purely classical overlapping interaction of the long-distance tails of the instanton fields, as in [7]. In the closed-string channel it can be interpreted as an off-shell propagator between Dirichlet boundary states

$$
\Gamma(x, y) \sim \sum_{s} C_{s}\left\langle B_{x}, s\left|\frac{1}{\Delta_{s}} P_{G S O}\right| B_{y}^{\prime}, s\right\rangle,
$$

where $C_{s}$ are appropriate phases for the coherent sum of spin structures in the GSOprojected closed-string channel, and $\Delta_{s}=\frac{\alpha^{\prime}}{2} p^{2}+N+\bar{N}-a_{s}-\bar{a}_{s}$ is the world-sheet Hamiltonian for type II strings. This static interaction vanishes for two instantons or two anti-instantons as a result of an unbroken supersymmetry in the open string channel (where we have a one-loop vacuum diagram of open strings with fixed endpoints, see [8] for explicit expressions of (1.1)). In the closed-string channel, this is just the well known "zero-force" property of BPS saturated configurations [9], [8], [2]. At the massless level, the 
cancellation results from the balance between the Coulomb interactions $\Gamma(x, y) \sim|x-y|^{-8}$, due to the attractive NS-NS dilaton exchange, and the repulsive R-R "axion" exchange. On the other hand, the $\mathrm{R}-\mathrm{R}$ scalar exchange becomes attractive between instantons and anti-instantons, a configuration that breaks all the supersymmetries:

$$
\Gamma_{+-}(x-y)=-(2 \pi)^{4} \int_{0}^{\infty} \frac{d s}{s^{5}} e^{-\frac{(x-y)^{2}}{2 \alpha^{\prime} s}} \prod_{m=1}^{\infty}\left(\frac{1+q^{2 m}}{1-q^{2 m}}\right)^{8}
$$

where $q=e^{-s}$ is the modular parameter of the cylindrical world-sheet. At short distances, this instanton/anti-instanton (I-A) interaction suffers from a Hagedorn singularity: each massive closed string contributing to (1.2) is suppressed by a factor $e^{-M|x-y|}$, and we can estimate the propagator as $\sum_{M} \rho_{D}(M) e^{-M|x-y|}$, where $\rho_{D}(M)$ is the level density of closed string states coupling to the D-instanton. This quantity grows exponentially with half the rate of the total density of states $\rho_{D}(M) \sim e^{\frac{\beta_{\mathrm{Hag}}}{2} M}$, which results in an instability at half the Hagedorn distance [10], [8]. Since (1.2) diverges at the ultraviolet endpoint $s \sim 0$, it is convenient to perform a modular transformation $t=2 \pi^{2} / s$ to the open-string channel, which is also useful to define the absolute normalization of the amplitude:

$$
\begin{aligned}
\Gamma_{+-}(x-y) & =-2 \cdot \frac{1}{4} \int_{0}^{\infty} \frac{d t}{t}\left[\operatorname{Tr}_{N S}\left(1+(-1)^{F}\right) e^{-t \Delta_{N S}}-\operatorname{Tr}_{R}\left(1+(-1)^{F}\right) e^{-t \Delta_{R}}\right] \\
& \left.=-\int_{0}^{\infty} \frac{d t}{t} e^{-t\left(\frac{(x-y)^{2}}{4 \pi^{2} \alpha^{\prime}}-\frac{1}{2}\right.}\right) \prod_{m=1}^{\infty}\left(\frac{1-w^{m-1 / 2}}{1-w^{m}}\right)^{8}
\end{aligned}
$$

where $w=e^{-t}$ and the overall factor of two is due to the oriented character of the type-IIB strings [2]. In what is by now a well understood rule [11], the open-string channel is the appropriate one to discuss short-distance dynamics of D-branes. Indeed, the massless states producing the $t \sim \infty$ singularities in (1.3) are associated to a vanishing Dirichlet openstring world-sheet Hamiltonian in the Neveu-Schwarz sector $\Delta_{N S}=\frac{(x-y)}{4 \pi^{2} \alpha^{\prime}}+N-\frac{1}{2}=0$, as a result of the balance between the Casimir and stretching energy of the Dirichlet open string. From (1.3) it follows that the dominant singularity occurs at half the Hagedorn distance of the type-IIB theory $\beta_{\mathrm{Hag}}=\pi \sqrt{8 \alpha^{\prime}}$, and it is logarithmically attractive: $\Gamma(x, y) \sim$ $\log \left(|x-y|-\beta_{\mathrm{Hag}} / 2\right)$.

An analogous singularity exists for higher $p$-branes, for which some derivative of the static force diverges [12]. These singularities indicate that I-A configurations are not appropriate varibles at string-length distances. Indeed, on general grounds, I-A configurations annihilate one another and cannot be distinguished from perturbative fluctuations 
in the coincidence limit, and their treatment is always very ambiguous at short distances. It is nevertheless surprising that a stringy instability poses a clear-cut limit to the I-A parametrization.

In the present paper we consider some effects on the I-A interaction due to supersymmetric zero modes or, equivalently, supersymmetric collective coordinates. Our main observation is that many familiar aspects of supersymmetric instanton calculus are recovered here from string world-sheet Ward identities.

Given the marginal character of the local I-A Hagedorn singularity (logarithmic), it is very interesting to check the effects of fermion zero modes at short distances. If the induced interactions turn out to be repulsive, they could perhaps cure the instability. In the course of this letter we will argue in favour of the opposite situation, namely the fermion-induced interactions are strongly attractive at short distances.

\section{Collective Coordinates}

In general, there is a fermionic collective coordinate for each fermionic symmetry broken by the classical solution. In the Dirichlet construction, we have to consider the space-time supersymmetries broken by the open-string boundary conditions. For explicit calculations involving the supersymmetry charges, it is convenient to use the light-cone Green-Schwarz formalism. In the notation and conventions of [8], the bosonic (anti-) Dinstanton boundary state is a solution of the constraints $\left(\alpha_{n}^{i}-\bar{\alpha}_{-n}^{i}\right)\left|I_{ \pm}, p\right\rangle=0,\left(S_{n}^{a} \pm\right.$ $\left.i \bar{S}_{-n}^{a}\right)\left|I_{ \pm}, p\right\rangle=0$ and may be written as the coherent state

$$
\left|I_{ \pm}, p\right\rangle=\exp \sum_{n=1}^{\infty}\left(\frac{\alpha_{-n}^{i} \bar{\alpha}_{-n}^{i}}{n} \mp i S_{-n}^{a} \bar{S}_{-n}^{a}\right)\left|0_{ \pm}, p\right\rangle
$$

where $S^{a}$ are Green-Schwarz fermions, transforming in the $\mathbf{8}_{\mathbf{s}}$ of $S O(8)$, the transverse rotation group. The ground states in (2.1) are the standard massless scalars of the type-IIB string $\left|0_{ \pm}, p\right\rangle=\frac{1}{4}(|p\rangle|i\rangle|\bar{i}\rangle \mp i|p\rangle|\dot{a}\rangle|\overline{\dot{a}}\rangle)$ satisfying $10_{ \pm}, p \mid=\left(\left|0_{\mp}, p\right\rangle\right)^{\dagger},\left\langle 0_{+}, p \mid 0_{+}, p^{\prime}\right\rangle=0$, $\left\langle 0_{+}, p \mid 0_{-}, p^{\prime}\right\rangle=\delta^{10}\left(p+p^{\prime}\right)$.

There are 32 supersymmetry charges in the type-IIB string. From the left-moving sector we have 16 charges with $S O(8)$ quantum numbers $\mathbf{8}_{\mathbf{s}} \oplus \mathbf{8}_{\mathbf{c}}$, with the $\mathbf{8}_{\mathbf{s}}$ charges given

1 As usual, the indices $i, a, \dot{a}$ run in the $\mathbf{8}_{\mathbf{v}}, \mathbf{8}_{\mathbf{s}}$ and $\mathbf{8}_{\mathbf{c}}$ of $S O(8)$ respectively. 
by $Q^{a}=\sqrt{2 p^{+}} S_{0}^{a}$, while those in the $\mathbf{8}_{\mathbf{c}}$ are non-linearly realized in the light-cone gauge, $Q^{\dot{a}}=\sqrt{\frac{2}{\alpha^{\prime} p^{+}}} \gamma_{\dot{a} a}^{i} \sum_{-\infty}^{+\infty} S_{-n}^{a} \alpha_{n}^{i}$, with the same structure repeated in the right-moving sector. The combinations $Q_{ \pm}=\frac{1}{\sqrt{2}}(Q \pm i \bar{Q})$ satisfy the algebra $\left\{Q_{+}, Q_{-}\right\}=\sqrt{2} \gamma_{\mu} p^{\mu}=$ $-i \sqrt{2} \gamma \cdot \partial$, after defining suitable $16 \times 16$ gamma matrices. The important property of the charges $Q_{ \pm}$is that they annihilate the instanton and anti-instanton: $Q_{ \pm}\left|I_{ \pm}, p\right\rangle=0$. Thus, the state $\left|I_{ \pm}\right\rangle$only breaks the $Q_{\mp}$ supersymmetries, and we have 16 fermionic collective coordinates in addition to the standard ten bosonic coordinates for the position (unlike Yang-Mills instantons, these $\mathrm{R}-\mathrm{R}$ gravitational instantons are "point-like", in the sense that no size parameter arises as a result of the lack of scale invariance).

These collective coordinates are introduced in the operator formalism by the insertion of operators:

$$
e^{i x P} e^{i \theta^{ \pm} Q_{\mp}}\left|I_{ \pm}, x=0\right\rangle
$$

for each instanton or anti-instanton boundary state of type (2.1) at fixed position. The measure for integration over the instanton moduli space must be invariant under the unbroken supertranslations $x \rightarrow x+\theta \gamma \theta$ and is thus determined to be

$$
d \mu_{ \pm}=J^{ \pm} d x^{ \pm} d^{16} \theta^{ \pm}
$$

with $J$ a convenient Jacobian, which can be obtained from the analysis of the low-energy solutions (see [13]). On general grounds, since the only bosonic zero modes are translations we know the scaling with the string coupling $\lambda$ as $J \sim\left(\sqrt{S_{c \ell}}\right)^{10} \sim \lambda^{-5}$, and the proportionality constant fixes the normalizations and gives the right dimensions to $d^{10} x_{0} d^{16} \theta$.

The action of $Q_{\mp}$ on $\left|I^{ \pm}\right\rangle$spans two $2^{16}$-dimensional supersymmetry representations. In particular, the standard fermionic zero modes correspond to the action of one charge $Q_{\mp}^{\alpha}\left|I^{ \pm}, x\right\rangle$, which has projections (wave functions) along any of the fermionic string states. Clearly, the integration over the fermionic collective coordinates is equivalent to including the complete D-instanton supermultiplet in the path integral. Following the rules in [5], [4], the partition function of the instanton gas is given by

$$
\mathcal{Z}=\sum_{n_{+}, n_{-}=0}^{\infty} \frac{1}{n_{+} ! n_{-} !} \prod_{j=1}^{n_{+}} \int d \mu_{j}^{+} \prod_{k=1}^{n_{-}} \int d \mu_{k}^{-} e^{-S_{\left(n_{+}, n_{-}\right)}}
$$

where the action in the $\left(n_{+}, n_{-}\right)$instanton sector is

$$
S_{\left(n_{+}, n_{-}\right)}=\Gamma_{0}+\sum_{j} \Gamma_{j}+\sum_{k} \Gamma_{k}+\sum_{\left(j_{1}, j_{2}\right)} \Gamma_{\left(j_{1}, j_{2}\right)}+\sum_{\left(k_{1}, k_{2}\right)} \Gamma_{\left(k_{1}, k_{2}\right)}+\sum_{(j, k)} \Gamma_{(j, k)}+3 \text { body; }
$$


here the index $j$ refers to instantons and $k$ to anti-instantons. We have an expansion in irreducible many-body interaction terms, each of them given by the sum of connected string diagrams with a number of boundaries attached to instantons, anti-instantons, or both. Specifically:

$$
\Gamma_{\left(j_{1}, \cdots ; k_{1} \cdots\right)}=\sum_{g=0}^{\infty} \sum_{N_{1}^{+}, \cdots=0}^{\infty} \sum_{N_{1}^{-}, \cdots=0}^{\infty} \frac{\lambda^{2 g+\sum N^{+}+\sum N^{-}-2}}{N_{1}^{+} ! \cdots N_{1}^{-} ! \cdots} W\left(g, N_{j}^{+}, N_{k}^{-}\right) .
$$

Here $N^{ \pm}$denote the number of boundaries attached to the same instanton or antiinstanton, and $\lambda$ stands for the string coupling constant. The first term $\Gamma_{0}$ is the standard perturbative sum of string diagrams in vacuo, and the bare instanton action is given by $\Gamma_{ \pm}=\frac{D}{\lambda}$, where $D$ is the disk amplitude. Diagrams with genus zero correspond to classical interactions between the instantons, the leading one coming from the cylinder diagram (1.1). The expansion (2.6) and (2.4) provides a complete perturbative treatment of the instanton interactions, including the purely classical ones, giving a stringy version of a perturbative constrained instanton expansion [14]. The BPS character ensures that there are no classical interactions between like- "ground-state" instantons, with boundary states given by (2.1). In fact, a heuristic non-renormalization theorem can be argued for the vanishing of the general string diagram with only (anti-) instanton insertions2. This is essentially Martinec's argument for the vanishing of the cosmological constant in perturbative string theory, using the corresponding unbroken supersymmetry. In principle, there are classical interactions between instanton states in the same supermultiplet, due to the fact that instantons do interact with anti-instantons, and both boundary states are related by the action of linearly realized supersymmetry charges: $\left|0_{ \pm}, p\right\rangle=\frac{1}{\left(2 p^{+}\right)^{4}} \prod_{a=1}^{8} Q_{ \pm}^{a}\left|0_{\mp}, p\right\rangle$. Therefore, the expansion in fermionic collective coordinates could effectively induce bosonic I-A interactions, as in (1.1). However, we shall see in the following that these terms do not survive the integration over fermionic collective coordinates.

In practice, the expressions (2.4) and (2.6) are only valid within a dilute-gas approximation, in spite of the systematic treatment of I-A interactions involved, and we are forced to cut-off the integral over positions at relative separations of the order of the string scale. The Hagedorn-like instability in the I-A sector is an obvious reason. However, even if we do not consider anti-instantons, the above parametrization of collective coordinates is

2 In fact, one needs at least one loop of closed or open strings to complete the argument, so that the disk amplitude is non-zero even in the supersymmetric case. 
wrong when several D-instantons approach one another. Below the string scale, we have $\sim N^{2}$ light modes instead of the $N$ positions of a set of $N$ D-instantons on top of each other [15], and the collective dynamics corresponds to a certain $U(N)$ supersymmetric matrix model (recently, the case $N=2$ was studied in [16]). A very interesting possibility along these lines would be that the effective dynamics of an I-A pair, separated by the singular distance $\beta_{\mathrm{Hag}} / 2$, is indeed described by some $U(2)$ matrix model.

\section{Ward Identity and Fermion Exchange}

It is clear that the previous setting can be used to obtain world-sheet Ward identities associated to the supersymmetries $Q_{ \pm}$. In order to generalize the discussion, it is convenient to use an abstract operator formalism in which the perturbative string amplitude with boundary states is represented as the overlap

$$
W\left(g, N_{j}^{+}, N_{k}^{-}\right)=\int_{\mathcal{M}}\left\langle\Sigma_{\left(g, N_{j}^{+}, N_{k}^{-}\right)} \mid \Psi\right\rangle,
$$

where we integrate over the moduli space of super-Riemann surfaces with punctures $\mathcal{M}$. The ket state has the structure $|\Psi\rangle=\otimes_{j}\left|x_{j}^{+}, \theta_{j}^{+}\right\rangle \otimes_{k}\left|x_{k}^{-}, \theta_{k}^{-}\right\rangle$and the states $\left|x^{ \pm}, \theta^{ \pm}\right\rangle=$

$e^{i \theta^{ \pm} Q_{\mp}}\left|x^{ \pm}\right\rangle$are one-punctured spheres, projected on the instanton boundary states located at the point $x^{ \pm}$; and we now allow the indices $j, k$ to label boundaries possibly located at the same space-time instanton. We will concentrate here on the multi-instanton vacuum amplitudes (2.6). However, it is clear that similar Ward identities could be derived for instanton-corrected scattering amplitudes, by simply including some asymptotic scattering states in the definition of the state $|\Psi\rangle$. See [17] [18] for some results in this direction.

The basic property we need is the contour-deformation formula to pull an insertion of a conserved current $Q=\oint J$ from one puncture to the others:

$$
\left.\langle\Sigma|Q| \psi\rangle \otimes_{i}\left|\chi_{i}\right\rangle=\sum_{i}\langle\Sigma \mid \psi\rangle \otimes\left|\chi_{1}\right\rangle \otimes \cdots \otimes|(-Q)| \chi_{i}\right\rangle \otimes \cdots
$$

and the exponentiated version: $\left\langle\Sigma \mid e^{Q} \psi\right\rangle \otimes_{i}\left|\chi_{i}\right\rangle=\langle\Sigma \mid \psi\rangle \otimes_{i} e^{-Q}\left|\chi_{i}\right\rangle$. These formal Ward identities hold up to total derivatives in the perturbative moduli space $\mathcal{M}$, which we tacitly discard. In doing so, our manipulations have the same heuristic status as the "proofs" of 
perturbative finiteness of superstrings. Applying the Ward identity to (3.1) we eliminate the fermionic collective coordinates from one puncture, say $x_{1}^{+}$, and we get

$$
\int_{\mathcal{M}}\left\langle\Sigma \mid x_{1}^{+}\right\rangle \otimes_{j \neq 1} e^{i\left(\theta_{j}^{+}-\theta_{1}^{+}\right) Q_{-}}\left|x_{j}^{+}\right\rangle \otimes_{k} e^{-i \theta_{1}^{+} Q_{-}} e^{i \theta_{k}^{-} Q_{+}}\left|x_{k}^{-}\right\rangle
$$

Now we can use the algebra $\left\{Q_{+}, Q_{-}\right\}=-i \sqrt{2} \gamma^{\mu} \partial_{\mu}$ to commute the two exponentials acting on the anti-instanton Hilbert spaces, at the expense of producing insertions of the momentum operator, which in turn translates the anti-instanton boundary states. The $Q_{\text {- }}$ charge annihilates the anti-instantons and we are left with

$$
\begin{gathered}
\int_{\mathcal{M}}\left\langle\Sigma \mid x_{1}^{+}\right\rangle \otimes_{j \neq 1} e^{i\left(\theta_{j}^{+}-\theta_{1}^{+}\right) Q_{-}}\left|x_{j}^{+}-i \sqrt{2} \theta_{1}^{-} \gamma\left(\theta_{j}^{+}-\theta_{1}^{+}\right)\right\rangle \otimes\left|x_{1}^{-}+i \sqrt{2} \theta_{1}^{-} \gamma \theta_{1}^{+}\right\rangle \\
\otimes_{k \neq 1} e^{i\left(\theta_{k}^{-}-\theta_{1}^{-}\right) Q_{+}}\left|x_{k}^{-}+i \sqrt{2} \theta_{k}^{-} \gamma \theta_{1}^{+}\right\rangle
\end{gathered}
$$

where we have applied the same manipulations to the $x_{1}^{-}$puncture. In the particularly simple case of an amplitude involving only instantons, the expression (3.4) reduces to $\int_{\mathcal{M}}\left\langle\Sigma \mid x_{1}^{+}\right\rangle \otimes_{j \neq 1}\left|x_{j}^{+}, \theta_{j}^{+}-\theta_{1}^{+}\right\rangle$, from which we can eliminate all $\theta_{1}$ dependence altogether by a change of variables in the measure: $\theta_{j} \rightarrow \theta_{j}+\theta_{1}$. Thus we see that the pure instanton vacuum amplitudes all vanish after integration over fermionic collective coordinates (again, up to total derivatives in the perturbative moduli space), even if, as stated before, the static interactions between some states in the supermultiplet could be non-trivial.

If $\theta_{j}^{+}=\theta_{1}^{+}$and $\theta_{k}^{-}=\theta_{1}^{-}$, i.e. when we have only one I-A pair connected by a Riemann surface, then we have succeeded in eliminating all fermionic collective coordinates in favour of a total derivative with respect to the relative position. This seems to be impossible for higher interactions ( 3 body, 4 body, etc). If we truncate the instanton action to the 2-body interaction terms, as in (2.5), then the partition function takes the form

$$
\begin{aligned}
\mathcal{Z}_{2-\text { body }}=e^{-\Gamma_{0}} \sum_{n_{+} n_{-}} \frac{1}{n_{+} ! n_{-} !} \int & \prod_{j} d x_{j}^{+} d \theta_{j}^{+} J_{j}^{+} e^{-\Gamma_{j}} \prod_{k} d x_{k}^{-} d \theta_{k}^{-} J_{k}^{-} e^{-\Gamma_{k}} \times \\
& \times \prod_{(j, k)} \exp \left(i \sqrt{2} \theta_{k}^{-} \gamma \theta_{j}^{+} \cdot \frac{\partial}{\partial x_{j k}}\right) e^{-\Gamma_{j k}\left(x_{j k}\right)}
\end{aligned}
$$

where $x_{j k}=x_{k}^{-}-x_{j}^{+}$and $\Gamma_{j k}\left(x_{j k}\right)$ is given by (1.2). Integration over the fermionic coordinates is equivalent to the insertion of factors $i \sqrt{2} \gamma_{\alpha \beta}^{\mu} \partial_{\mu} \Gamma_{2}\left(x^{-}-x^{+}\right)$for each pairing of fermionic lines, in a graphical representation in which each instanton is an effective operator with 16 fermionic legs, labelled by the $\mathbf{8}_{\mathbf{s}} \oplus \mathbf{8}_{\mathbf{c}}$ of $S O(8)$ Dirac indices. In eq. (1.1), $\Gamma_{2}\left(x^{-}{ }_{-}\right.$ 
$x^{+}$) was interpreted as an off-shell tree-level propagator (inverse generalized Laplacian) for the bosonic boundary states, thus the fermionic insertions are true off-shell string field theory generalizations of fermionic propagators. Notice that the function $\Gamma_{2}\left(x^{-}-x^{+}\right)$ may contain contributions from an arbitrary number of boundaries and handles, so that we really obtain the fully dressed fermionic propagators of string field theory.

We can also saturate the zero modes by applying several derivatives to the same offshell propagator. A term with an even number of derivatives $(\gamma \cdot \partial)^{2 n} \Gamma_{2}$ can be interpreted as a bosonic line connecting effective operators with $n$ derivatives $\mathcal{O}_{b} \sim \varphi^{N_{b}} \partial^{n} \psi^{N_{f}}$, with an even number of fermions $N_{f}=$ even. On the other hand, terms with an odd number of derivatives $(\gamma \cdot \partial)^{2 n+1} \Gamma_{2}$ may be interpreted as a fermionic propagator connecting fermionic operators with $n$ derivatives $\mathcal{O}_{f} \sim \varphi^{N_{b}} \partial^{n} \psi^{N_{f}}$, and $N_{f}=$ odd. We thus get the stringy generalization of the standard rules for the clustering of zero modes, and the multiplescattering approximation familiar from Field Theory [19]. It is very satisfying to see them emerge from world-sheet Ward identities in the underlying string theory.

We can exhibit the effective operators more precisely in the one-instanton sector. Fermionic zero modes pose selection rules on the effective operators arising in the process of "integrating out" instanton fluctuations. In the one-instanton sector we have to saturate the 16 zero modes of the D-instanton, and the analogue of the resulting 't Hooft effective interaction is generated to leading order in the string coupling by 16 tadpoles of fermionic vertex operators $V^{\psi}$ in the instanton background. This easily follows from the fermionic D-instanton calculus in the form

$$
\int d x_{0} d \theta J e^{-\Gamma_{1}} \prod_{\alpha=1}^{16}\left\langle V_{p_{\alpha}}^{\psi}\left|e^{i \theta^{ \pm} Q_{\mp}}\right| I^{ \pm}, x_{0}\right\rangle_{\text {disk }}=\int d x_{0} J e^{-\Gamma_{1}} \prod_{\alpha=1}^{16}\left\langle V_{p_{\alpha}}^{\psi}\left|Q_{\mp}^{\alpha}\right| I^{ \pm}, x_{0}\right\rangle_{\text {disk }} .
$$

In general, string scattering amplitudes directly produce the amputated Green function of string field theory. Accordingly, the Dirichlet disk tadpoles can be interpreted as the source terms in the classical background equations through the formal relation

$$
\left\langle V_{\Phi}(p) \mid I, x_{0}\right\rangle_{\text {disk }} \sim\left(p^{2}+M_{\Phi}^{2}\right)\langle\Phi(p)\rangle_{I, x_{0}} .
$$

At the massless level, this simply reduces to $\partial_{x}^{2} \varphi_{c \ell}\left(x, x_{0}\right)$, where $\varphi_{c \ell}\left(x, x_{0}\right)$ denotes collectively the bosonic massless fields in the background of an instanton located at $x_{0}$. These are particularly simple in the case of the type-IIB gravitational instantons we are interested 
in: at the massless level [6], the D-instanton only couples to the dilaton $\phi$ and the $\mathrm{R}-\mathrm{R}$ scalar "axion"

$$
i a_{c \ell} \sim e^{-\phi_{c \ell}}=e^{-\phi_{\infty}}\left(1+\frac{Q \alpha^{4}}{\left|x-x_{0}\right|^{8}}\right)^{-1}
$$

in the bosonic sector $\mathrm{B}$, where $Q$ denotes the $\mathrm{R}-\mathrm{R}$ charge in appropriate units. The fermionic zero modes $\gamma \cdot D \psi_{c \ell}=0$ appearing in (3.6) are obtained from the bosonic solution by a broken supersymmetry transformation. In general, starting from the bosonic solution we can generate the full fermionic instanton solution as a function of both bosonic and fermionic collective coordinates (i.e. the full supermultiplet of instanton fields) by explicit iteration of the type-IIB supersymmetry transformations as presented in [20]. Formally, one finds the structure

$$
\begin{aligned}
& \varphi_{c \ell}\left(x, x_{0}, \theta\right) \sim \sum_{n=0}^{8} C_{n}\left(\theta \gamma \cdot \partial_{x} \theta\right)^{n} \varphi_{c \ell}\left(x, x_{0}\right) \\
& \psi_{c \ell}\left(x, x_{0}, \theta\right) \sim \sum_{n=0}^{7} C_{n}\left(\theta \gamma \cdot \partial_{x} \theta\right)^{n} \theta \gamma \cdot \partial_{x} \varphi_{c \ell}\left(x, x_{0}\right)
\end{aligned}
$$

for bosonic and fermionic fields respectively. Now, all effective operators produced by integrating out D-instantons are generated in the local limit by the standard rule:

$$
\left\langle\prod_{\text {bosons }} \partial^{2} \varphi \prod_{\text {fermions }} \gamma \cdot \partial \psi\right\rangle \rightarrow \sum_{\text {inst }}\left\langle\mathcal{O}_{\text {eff }}(\varphi, \psi)\right\rangle_{\text {vac }} .
$$

Here the factors $\partial^{2}$ and $\gamma \cdot \partial$ correspond to the amputation of the Green function. The right-hand side of (3.8) has the form

$$
\mathcal{O}_{\text {eff }} \sim e^{-D / \lambda} J A \varphi^{N_{b}} \partial^{N_{\partial}} \psi^{N_{f}}
$$

for an effective operator with $N_{b}$ bosons, $N_{f}$ fermions and $N_{\partial}$ derivatives. Here $A$ denotes the contribution of non-zero modes (starting with the one-loop determinant in the instanton background).

More explicitly, the effective operators are given by all the different forms of saturating the fermionic integration in the expression

$$
e^{-\Gamma_{1}} \int d x_{0} d^{16} \theta J \prod_{j=1}^{N_{b}} \partial_{x_{j}}^{2} \varphi_{c \ell}\left(x_{j}, x_{0}, \theta\right) \prod_{k=1}^{N_{f}} \gamma \cdot \partial_{x_{k}} \psi_{c \ell}\left(x_{k}, x_{0}, \theta\right),
$$

3 This is an elementary solution of the Laplace equation with source at $x_{0}$, so $\varphi_{c \ell}\left(x, x_{0}\right) \sim$ $\left\langle x_{0}\left|\frac{1}{\partial^{2}}\right| x\right\rangle$ indeed scales like a massless bosonic propagator. 
where $\varphi\left(x, x_{0}, \theta\right)$ and $\psi\left(x, x_{0}, \theta\right)$ are the classical configurations in (3.7), in complete analogy with the superfield instanton calculus as developed for example in [21]. If each bosonic leg soaks up $2 n_{j}$ fermion collective coordinates, and each fermionic leg $2 m_{k}+1$ such that $\sum 2 n_{j}+\sum\left(2 m_{k}+1\right)=16$, then, considering the amputation factors and the scaling of the bosonic solution $\varphi_{c \ell} \sim(\partial)^{-2}$, we find a total of $\sum n_{j}+\sum m_{k}=8-\frac{N_{f}}{2}$ derivatives in the effective operator. In general, defining the natural dimensional index for supersymmetric transformations $\mathcal{N} \equiv N_{\partial}+N_{f} / 2$, we have the selection rule $\mathcal{N}=\frac{Z}{2}$ for $Z$ chiral zero modes.

Realizing the supersymmetry in the linearized approximation, there are additional selection rules coming from the BPS character of the instantons, and the fact that the only bosonic massless couplings correspond to the two scalars of the massless type-IIB multiplet. The discussion is simplified by the fact that we may arrange the massless IIB supermultiplet into a light-cone superfield containing the antisymmetric products of the $\mathbf{8}_{\mathbf{s}}$ representation of $S O(8)$ according to ref. [22]. In any case, from the physical point of view, the upshot of this discussion of zero-mode selection rules is that effective operators induced by ten-dimensional D-instantons of the type-IIB theory have 16 fermions or an equivalent number of derivatives. They are thus of very high dimension, and no interesting infrared dynamics is induced in the one-instanton sector. This is true in spite of the strong Coulomb forces binding the instantons and anti-instantons, similar to the familiar case of $2+1$ gauge theories [23], [24]. This is consistent with the fact that supersymmetry completely determines the type-IIB supergravity action to order $\mathcal{N}=2$, and no potential is allowed. On general grounds, since the instanton "dynamics" reduces to a finite-dimensional integral, we expect the effective action after integrating out the instanton fluctuations to be supersymmetric. This means that one expects instantons to induce effective actions that could exhibit spontaneous breaking of supersymmetry in some cases. On the other hand a non-supersymmetric effective action after integrating out instantons would indicate that supersymmetry is anomalous. As we comment below, a possible exception could be the I-A sector, because of the Hagedorn singularity.

On the other hand, working in less supersymmetric backgrounds, D-instantons or more generally wrapped Euclidean D-branes could indeed generate interesting couplings in the infrared. A notorious example appears in [25], where superpotentials are generated in $N=1$ backgrounds. An example with completely broken supersymmetry is given by the finite-temperature boundary conditions, where one readily finds periodic potentials 
for the ten-dimensional axion field, and runaway potentials for the dilaton generated by D-instantons (see [13]).

Coming back to (3.5), we can discuss the dynamical effects of including the fermionic collective coordinates. At large separations, in the dilute-gas limit, we obtain a supressing factor $\left|x^{+}-x^{-}\right|^{-9}$ for each paired fermion line, from the long-distance scaling of the fermionic propagator $\gamma \cdot \partial \Gamma_{2}\left(x^{+}-x^{-}\right)$. This corresponds to the restoration of supersymmetric zero modes (for example, factorization of determinants at one loop), and the subsequent suppression of instanton effects.

At string-scale distances, we can estimate the fermionic propagators in the open channel expression (1.3). Each pair of fermionic collective coordinates carries a derivative insertion and therefore an extra enhancement factor $\left|x^{+}-x^{-}-\beta_{\mathrm{Hag}} / 2\right|^{-1}$ in the partition function (a logarithmic attraction term in the effective action). This means that fermion exchange is also strongly attractive at short distances. We may consider as possible dominant configurations those in which the fermionic zero modes are saturated in "dipole" pairs, each one contributing an extra factor $\left|x^{+}-x^{-}-\beta_{\mathrm{Hag}} / 2\right|^{-16}$. These configurations are also interesting because they integrate to a total derivative in the multi I-A partition function. Indeed, summing a dilute gas of these "dipoles", we obtain a vacuum effective action

$$
\Gamma_{\text {dipoles }}=\Gamma_{0}+\operatorname{Vol}_{10} \times e^{-2 \Gamma_{1}} \times 2^{8} \int_{0}^{\infty} d x \operatorname{det}\left(\gamma \cdot \partial_{x}\right) e^{-\Gamma_{2}(x)},
$$

where the determinant is over Dirac indices in the $\mathbf{8}_{\mathbf{s}} \oplus \mathbf{8}_{\mathbf{c}}$ of $S O(8)$. In a normal situation, as in field theory, one would define the $\mathrm{I}-\mathrm{A}$ coincidence boundary condition at $x=0$ as trivial, thereby obtaining $\Gamma_{\text {dipoles }}=0$ and unbroken supersymmetry. In this case, the logarithmic singularity at finite separation renders (3.11) ill defined and there is a possibility of supersymmetry breaking, depending on the boundary conditions (i.e. new physics) involving the annihilation of the I-A pairs, which might signal an interesting source of non-perturbative instabilities of type-IIB strings.

In any case, it is clear that no definite conclusions can be drawn until the physics of I-A annihilation is understood. In fact, we can view the singularity as an effect of not treating the extra massless modes appearing at the distance $\beta_{\mathrm{Hag}} / 2$ as fully fledged collective coordinates. Therefore, one should perhaps formulate the problem in terms of the $U(2)$ matrix dynamics of light modes of I-A "molecules" of approximate size $\beta_{\mathrm{Hag}} / 2$. However, the study of possible bound states of the resulting I-A system is surely complicated by the lack of supersymmetry of these configurations. 


\section{Conclusions}

We observe that the stringy generalization of some standard rules of instanton calculus is simply dictated by the structure of world-sheet Ward identities. This is the case of the handling of supersymmetry zero modes and the corresponding fermion exchange interactions. The consideration of anti-commuting collective coordinates does not resolve the Hagedorn instability of overlapping I-A pairs. On the contrary, the interaction induced by fermion exchange is strongly attractive at the string-scale distances.

Ten-dimensional D-instantons induce effective operators with $N_{\partial}+\frac{N_{f}}{2}=8$, where $N_{\partial}$ is the number of derivatives and $N_{f}$ is the number of fermions. Therefore, they are not very important in the infrared unless the I-A strong dynamics somehow breaks supersymmetry. It is interesting to consider explicit D-instanton constructions in four-dimensional $N=1$ backgrounds. In this case, in addition to the ten-dimensional D-instantons, we would have wrapped Euclidean D-strings, 3-branes and 5-branes, as in [26]. Four dimensional superpotentials would be generated if the instanton has exactly two chiral fermion zero modes. A very general characterization of such superpotentials was recently put forward in [25]. It would be interesting to work out in more detail the weak coupling quantization of such four-dimensional $\mathrm{R}-\mathrm{R}$ instantons.

Much work is still needed in order to fully understand the collective dynamics of D-instantons and D-branes in general. The occurrence of a Hagedorn singularity in nonsupersymmetric sectors may indicate that new surprises beyond the $U(N)$ enhancement phenomenon of [15] are possible. It is then very important to learn the semiclassical rules that reproduce non-trivial exact results such as [27].

\section{Acknowledgements}

It is a pleasure to thank E. Alvarez, L. Alvarez-Gaumé, C. Gómez, M.A. VázquezMozo, and specially D.J. Gross and I.R. Klebanov for useful discussions and suggestions. Note added in print: The recent preprint 28 has some overlap with our treatment. 


\section{References}

[1] J. Dai, R.G. Leigh and J. Polchinski, Mod. Phys. Lett. A4 (1989) 2073.

[2] J. Polchinski, Phys. Rev. Lett. 75 (1995) 4728, hep-th/9510017.

[3] E.F. Corrigan and D.B. Fairlie, Nucl. Phys. B91 (1975) 527;

M.B. Green, Phys. Lett. B69 (1976) 89;

I.R. Klebanov and L. Thorlacius, Phys. Lett. B371 (1996) 51, hep-th/9510200;

J.L.F. Barbón, Phys. Lett. B382 (1996) 60, hep-th/9601098.

[4] M.B. Green, Phys. Lett. B354 (1995) 271, hep-th/9504108.

[5] J. Polchinski, Phys. Rev. D50 (1994) 6041, hep-th/9407031.

[6] G.W. Gibbons, M.B. Green and M.J. Perry, Phys. Lett. B370 (1996) 37, hepth/9511080.

[7] C.G. Callan, R. Dashen and D.J. Gross, Phys. Rev. D17 (1978) 2717.

[8] M.B. Green, Phys. Lett. B329 (1994) 435, hep-th/9403040.

[9] C. Montonen and D. Olive, Phys. Lett. B72 (1977) 117.

[10] M.B. Green, Phys. Lett. B266 (1991) 325.

[11] M.R. Douglas, D. Kabat, P. Pouliot and S. Shenker, RU-96-62, hep-th/9608024.

[12] T. Banks and L. Susskind, RU-95-87, hep-th/9511194.

[13] J.L.F. Barbón and M.A. Vázquez-Mozo, CERN-TH/96-361, IASSNS-96/127, hepth/9701142.

[14] I. Affleck, Nucl. Phys. B191 (1981) 429.

[15] E. Witten, Nucl. Phys. B460 (1996) 335, hep-th/9510135.

[16] M.B. Green and M. Gutperle, DAMTP-96-110, hep-th/9612127.

[17] M.B. Green and M. Gutperle, Nucl. Phys. B476 (1996) 484, hep-th/9604091.

[18] K. Hamada, KEK-TH-504, hep-th/9612234.

[19] E. Mottola, Phys. Rev. D17 (1978) 1103.

[20] J.H. Schwarz, Nucl. Phys. B226 (1983) 269.

[21] V.A. Novikov, M.A. Shifman, A.I. Vainshtein, V.B. Voloshin and V.I. Zakharov, Nucl. Phys. B229 (1983) 394.

[22] M.B. Green and J.H. Schwarz, Phys. Lett. B122 (1983) 143.

[23] A.M. Polyakov, Nucl. Phys. B120 (1977) 429.

[24] I. Affleck, J. Harvey and E. Witten, Nucl. Phys. B206 (1982) 413. 
[25] E. Witten, Nucl. Phys. B474 (1996) 343, hep-th/9604030.

[26] K. Becker, M. Becker and A. Strominger, Nucl. Phys. B456 (1995) 130, hepth/9507158.

[27] H. Ooguri and C. Vafa, HUTP-96-A036, hep-th/9608079.

[28] M.B. Green and M. Gutperle, DAMTP/96-104, hep-th/9701093. 